(1)

CrossMark

\title{
Mast cells in asthma: Here I am, stuck in the middle with you
}

\author{
Raymond B. Penn
}

Affiliation: Dept of Medicine, Division of Pulmonary and Critical Care Medicine, Center for Translational Medicine, Jane and Leonard Korman Respiratory Institute, Thomas Jefferson University, Philadelphia, PA, USA.

Correspondence: Raymond B. Penn, Center for Translational Medicine, Thomas Jefferson University, Rm 543 JAH, 1020 Locust St., Philadelphia, PA, 19107, USA; E-mail: raymond.penndjefferson.edu

@ERSpublications

Bonvini and co-workers employ a creative combination of cell, tissue and in vivo models to delineate a complex intercellular communication between mast cells and airway smooth muscle by which TRPV4 activation causes bronchoconstriction https://bit.ly/35U4Mqg

Cite this article as: Penn RB. Mast cells in asthma: Here I am, stuck in the middle with you. Eur Respir J 2020; 56: 2001337 [https://doi.org/10.1183/13993003.01337-2020].

Mast cells play an important role in allergic lung inflammation in asthma, contributing to both the early-phase reaction as well as the late-phase reactions of asthma through the release of numerous agents [1]. Mast cell precursors from the blood migrate to and embed themselves into the airway wall, including within airway smooth muscle (ASM) bundles $[2,3]$, where they are exposed to a host of local factors that cause them to "mature" into a critical immunomodulatory cell [4]. Cromylyn sodium, based on its presumed ability as a "mast cell stabiliser", was once a popular asthma drug (and preferred for children), before corticosteroids and cysteinyl leukotriene (CysLT) receptor antagonists emerged as the drugs of choice for managing lung inflammation [5].

In addition to coordinating the progression, nature and resolution of allergic inflammation in the asthmatic lung, mast cells can directly promote ASM contraction and bronchoconstriction by releasing agents (e.g. CysLTs, histamine) that serve as contractile agonists. The best-characterised mechanism by which mast cells secrete pro-contractile agonists is via activation of the high affinity immunoglobulin $\mathrm{E}$ (IgE) receptor, FceRI. Airway IgE levels are elevated during allergic lung inflammation associated with asthma, causing mast cell FceRI activation and release of contractile agonists (primarily CysLTs and histamine in human mast cells) that cause ASM contraction and thus bronchoconstriction (as reviewed previously [6]). This in vivo effect can be deduced from multiple rodent and human studies, and can be validated in cell- and tissue-based studies. Airway contraction evidenced in human precision cut lung slices (PCLS) caused by FceRI crosslinking was partially inhibited by either the CysLT1 receptor antagonist montelukast or the antihistamine fexofenadine, whereas inhibition of spleen tyrosine kinase Syk (which is absent in human ASM) effectively ablated the contraction while almost totally blocking the FceRI-mediated release of histamine and CysLTs [7].

IgE/FceRI-independent mechanisms of mast cell activation also exist and can mediate bronchoconstriction, although such mechanisms are not as well-delineated as those involving IgE/FceRI. One mechanism of ASM contraction speculated to utilise IgE/FceRI-independent mast cell activation involves transient receptor potential vanilloid 4 (TRPV4). TRPV4, a calcium-permeable cation channel, can be activated by a wide range of stimuli, including osmotic stress, low $\mathrm{pH}$, arachidonate products, mechanical stimuli and heat [8]. TRPV4 is widely expressed (although not on mast cells) and has been 
well-characterised with respect to mechanisms mediating pain and oedema. It is also an intriguing regulator of airway function. TRPV4 plays a role in cough, as TRPV4 agonists depolarise the human vagus nerve and stimulate $\mathrm{A} \delta$ fibres, and cause cough in conscious guinea pigs, all effects blocked by a TRPV4 antagonist [9]. TRPV4 is also expressed on ASM, and TRPV4 agonism has been shown to increase ASM intracellular calcium [10] and cause contraction of guinea pig and human airways ex vivo [11]. MCALEXANDER et al. [11] demonstrated the airway contraction by TRV4 agonism to be entirely dependent on CysLTs, being blocked by the CysLT type 1 receptor antagonist montelukast, but given the lack of any published evidence linking TRV4 and CysLT production, how TRPV4 on ASM could cause CysLT-dependent contraction remained a puzzle.

Trying to make some sense of it all, Bonvini et al. [12] employed a creative combination of cell, tissue and in vivo models to delineate a complex intercellular communication by which TRPV4 activation causes bronchoconstriction. After establishing the ability of TRPV4 agonist GSK1016790A to increase airway resistance in conscious guinea pigs, the pro-contractile effect of GSK1016790A on ASM was demonstrated in organ bath studies using tracheal tissue preparations isolated from both guinea pig and human lung. These in vivo and ex vivo effects were all inhibited by pretreatment with the TRPV4 antagonist GSK2193874. Consistent with the findings of MCAlEXANDER et al. [11], both CysLT1R antagonism (montelukast) as well as 5-LO inhibition (zileuton) inhibited the TRPV4-stimulated contraction of human ASM tissue, confirming the role of CysLTs. In cultured human ASM cells, GSK1016790A increased intracellular calcium. To assess the capacity of this induction of calcium to promote ASM contraction, the authors employed a novel imaging technique, ptychography. Ptychography is a label-free microscopy technique that analyses three-dimensional characteristics of the cell to provide a measure of cell contraction concomitant with assessment of changes in intracellular calcium. Somewhat surprisingly, ptychography revealed that the GSK1016790A-induced increase in intracellular calcium did not cause cell contraction in cultured ASM. Because human mast cells lack TRPV4, these results suggested that activation of TRV4 on human ASM causes the release of a mediator that activates mast cells.

Subsequent experiments demonstrated GSK1016790A stimulated ATP release from human ASM cells in culture. Exogenous ATP could be shown to stimulate contraction of human ASM tissue in organ bath studies, and this effect was blocked by both montelukast and zileuton. Using a battery of subtype-selective purinergic receptor antagonists, the pro-contractile effect of GSK1016790A in co-cultures on human ASM and mast cells, and in human airway tissue, was inhibited only by the P2X4 receptor antagonist 5BDBD.

A final set of experiments sought to establish the physiological/pathophysiological relevance of this elegant intercellular cross-talk mechanism (figure 1). Because protease-activated receptor 2 (PAR2) is known to couple to and gate TRPV4 [13], and also be expressed on human ASM [10], the authors tested the ability of the endogenous PAR2 agonist trypsin to replicate the effect of GSK1016790A. Indeed, trypsin

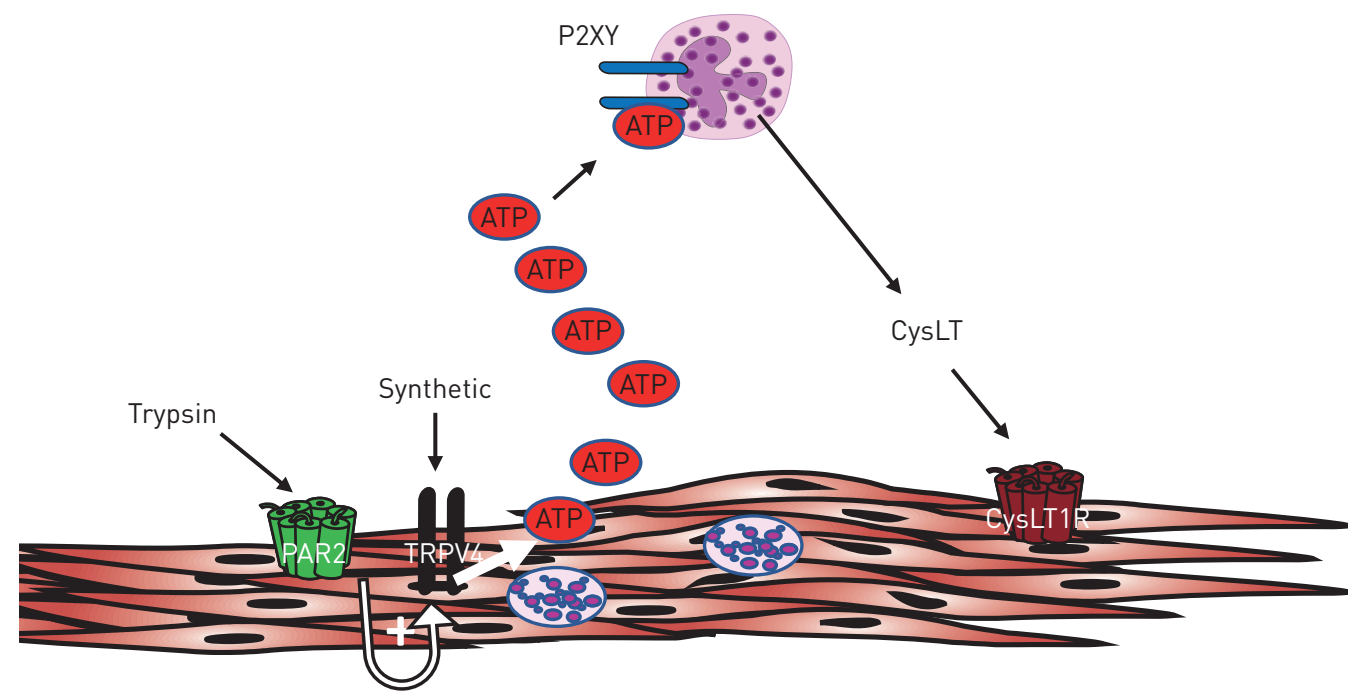

FIGURE 1 Transient receptor potential vanilloid 4 (TRPV4)-mediated airway smooth muscle (ASM) contraction via mast cell-ASM crosstalk. TRV4 channels on ASM are gated either directly via synthetic agonists or via ASM protease-activated receptor 2 (PAR2) activation. TRV4 activation results in ATP secretion by ASM, activation of mast cell P2X4 receptor and release of cysteinyl leukotrienes (CysLTs) by mast cells embedded within the airway. The paracrine/juxtacrine CysLTs activate CysLT1 receptors (CysLT1R) on ASM, resulting in ASM contraction. 
stimulated contraction of human ASM tissue was blocked by GSK2193874, montelukast and 5BDBD, as well as by the serine protease inhibitor camostat mesylate.

The findings of Bonvini et al. [12] provide important insight into the role of the mast cell in allergen-independent processes capable of causing bronchoconstriction in diseases such as exercise-induced asthma, in which bronchoconstriction is believed to be caused by hyperosmolarity/ thermal changes of the airway lining associated with ventilation of cool, dry air [14]. In addition, one could also speculate that this same PAR2/TRPV4/ATP/P2X4/CysLT axis could play a role in bronchoconstriction associated with fungal infection/colonisation of the lung, given the known ability of protease activity in certain fungi (e.g. Alternaria alternata) to activate PAR2 [15]. Gastro-oesophageal reflux disease, or microaspiration of acid from various sources, could also activate TPRV4 on ASM to induce bronchoconstriction. And although many of these conditions also possess an allergic component capable of allergen activation of mast cells, it is conceivable that a TPRV4-dependent allergen-independent mechanism could act in concert with allergen-dependent activation of mast cells to affect the initiation, magnitude and duration of mast cell effects.

From a more basic science perspective, the finding that TRPV4-mediated calcium increases in ASM fail to directly promote cell contraction, as originally assumed by JiA et al. [10], provides further evidence that compartmentalisation of calcium dictates the functional consequences of calcium. Whereas historically, increases in intracellular calcium in ASM cells have been reflexively interpreted as a procontractile signal, there are now numerous examples in which this is clearly not the case. In some instances, increases in intracellular calcium appear to cause relaxation of ASM. In the case of ASM bitter tastant receptor activation [16], intracellular calcium increases relax ASM likely via intracellular actions involving either actin cytoskeleton depolymerisation [17], gating of large-conductance $\mathrm{Ca}^{2+}$-activated $\mathrm{K}^{+}\left(\mathrm{BK}_{\mathrm{Ca}}\right)$ channels [16], or the regulation of other intercellular calcium pools that regulate calcium-dependent cross bridge cycling $[18,19]$. In the case of BK1 bradykinin receptor and possibly the proton-sensing receptor OGR1/ GPR68, calcium-dependent signalling linked to PKC activation and cyclooxygenase activation produces prostanoids that act in an autocrine manner to relax ASM via PKA-activating EP/IP receptors [20-23]. In the case of TRPV4 effects on ASM, calcium signalling is uncoupled from (direct) regulation of contraction, causing ASM to function instead as an exocrine cell.

Lastly, the establishment of this novel intracellular crosstalk mechanism mediating ASM contraction further advances TRPV4 as an important regulatory molecule in lung physiology. As noted above, TRPV4 is activated by diverse stimuli, and has been implicated in cough, asthma, COPD, pulmonary fibrosis, pulmonary hypertension, lung oedema, and protection from bacterial pneumonia and ventilation-induced lung injury (as reviewed previously [8]). The growing literature implicating TRPV4 in multiple disease processes strongly suggests a future role for therapeutic agents targeting TRPV4.

Conflict of interest: R.B. Penn has nothing to disclose.

Support statement: R.B. Penn's research is funded by NIH grants R01HL136209, R01AI110007, R01 HL058506, P01 HL114471, and R01HL145392. Funding information for this article has been deposited with the Crossref Funder Registry.

\section{References}

Galli SJ, Tsai M. IgE and mast cells in allergic disease. Nat Med 2012; 18: 693-704.

2 Begueret H, Berger P, Vernejoux JM, et al. Inflammation of bronchial smooth muscle in allergic asthma. Thorax 2007; 62: 8-15.

3 Brightling CE, Bradding P, Symon FA, et al. Mast-cell infiltration of airway smooth muscle in asthma. N Engl J Med 2002; 346: 1699-1705.

4 Dahlin JS, Hallgren J. Mast cell progenitors: origin, development and migration to tissues. Mol Immunol 2015; 63: 9-17.

5 van der Wouden JC, Uijen JH, Bernsen RM, et al. Inhaled sodium cromoglycate for asthma in children. Cochrane Database Syst Rev 2008: CD002173.

$6 \quad$ Bradding P, Arthur G. Mast cells in asthma-state of the art. Clin Exp Allergy 2016; 46: 194-263.

7 Koziol-White CJ, Jia Y, Baltus GA, et al. Inhibition of spleen tyrosine kinase attenuates IgE-mediated airway contraction and mediator release in human precision cut lung slices. Br J Pharmacol 2016; 173: 3080-3087.

8 Grace MS, Bonvini SJ, Belvisi MG, et al. Modulation of the TRPV4 ion channel as a therapeutic target for disease. Pharmacol Ther 2017; 177: 9-22.

9 Bonvini SJ, Birrell MA, Grace MS, et al. Transient receptor potential cation channel, subfamily V, member 4 and airway sensory afferent activation: role of adenosine triphosphate. J Allergy Clin Immunol 2016; 138: 249-261.

10 Jia Y, Wang X, Varty L, et al. Functional TRPV4 channels are expressed in human airway smooth muscle cells. Am J Physiol Lung Cell Mol Physiol 2004; 287: L272-L278.

11 McAlexander MA, Luttmann MA, Hunsberger GE, et al. Transient receptor potential vanilloid 4 activation constricts the human bronchus via the release of cysteinyl leukotrienes. J Pharmacol Exp Ther 2014; 349: 118-125. 
12 Bonvini SJ, Birrell MA, Dubuis E, et al. Novel airway smooth muscle-mast cell interactions and a role for the TRPV4-ATP axis in non-atopic asthma. Eur Respir J 2020; 56: 1901458.

13 Poole DP, Amadesi S, Veldhuis NA, et al. Protease-activated receptor 2 (PAR2) protein and transient receptor potential vanilloid 4 (TRPV4) protein coupling is required for sustained inflammatory signaling. J Biol Chem 2013; 288: 5790-5802.

14 Kippelen P, Anderson SD, Hallstrand TS. Mechanisms and biomarkers of exercise-induced bronchoconstriction. Immunol Allergy Clin North Am 2018; 38: 165-182.

15 Boitano S, Flynn AN, Sherwood CL, et al. Alternaria alternata serine proteases induce lung inflammation and airway epithelial cell activation via PAR2. Am J Physiol Lung Cell Mol Physiol 2011; 300: L605-L614.

16 Deshpande DA, Wang WC, McIlmoyle EL, et al. Bitter taste receptors on airway smooth muscle bronchodilate by localized calcium signaling and reverse obstruction. Nat Med 2010; 16: 1299-1304.

17 Tazzeo T, Bates G, Roman HN, et al. Caffeine relaxes smooth muscle through actin depolymerization. Am J Physiol Lung Cell Mol Physiol 2012; 303: L334-L342.

18 Nayak AP, Shah SD, Michael JV, et al. Bitter taste receptors for asthma therapeutics. Front Physiol 2019; 10: 884.

19 Talmon M, Rossi S, Lim D, et al. Absinthin, an agonist of the bitter taste receptor hTAS2R46, uncovers an ER-to-mitochondria $\mathrm{Ca}^{2+}$-shuttling event. J Biol Chem 2019; 294: 12472-12482.

20 Belvisi MG, Saunders M, Yacoub M, et al. Expression of cyclo-oxygenase-2 in human airway smooth muscle is associated with profound reductions in cell growth. Br J Pharmacol 1998; 125: 1102-1108.

21 Michael JV, Gavrila A, Nayak AP, et al. Cooperativity of E-prostanoid receptor subtypes in regulating signaling and growth inhibition in human airway smooth muscle. FASEB J 2019; 33: 4780-4789.

22 Nayak AP, Pera T, Deshpande DA, et al. Regulation of ovarian cancer G protein-coupled receptor (OGR1) expression and signaling. Am J Physiol Lung Cell Mol Physiol 2019; 316: L894-L902.

23 Pang L, Knox AJ. PGE2 release by bradykinin in human airway smooth muscle cells: involvement of cyclooxygenase-2 induction. Am J Physiol 1997; 273: 1132-1140. 